\title{
Crystal structure of $A_{3} B_{3}$ complex of V-ATPase from Thermus thermophilus
}

This is an open-access article distributed under the terms of the Creative Commons Attribution License, which permits distribution, and reproduction in any medium, provided the original author and source are credited. This license does not permit commercial exploitation without specific permission.

\section{Megan J Maher 1,8,9, Satoru Akimoto ${ }^{2,8}$, Momi Iwata ${ }^{1,3,8}$, Koji Nagata ${ }^{1}$, Yoshiko Hori $^{2}$, Masasuke Yoshida 4,5 , Shigeyuki Yokoyama ${ }^{2}$, So Iwata ${ }^{1,3,6,7, *}$ and Ken Yokoyama ${ }^{2,4,5, *}$}

\begin{abstract}
${ }^{1}$ Division of Molecular Biosciences, Imperial College London, South Kensington Campus, London, UK, ${ }^{2}$ Protein Research Group, Genomic Sciences Center, Yokohama Institute, RIKEN, 1-7-22 Suehiro-cho, Tsurumi-ku, Yokohama, Japan, ${ }^{3}$ Membrane Protein Laboratory, Diamond Light Source Limited, Harwell Science and Innovation Campus, Chilton, Didcot, Oxfordshire, UK, ${ }^{4}$ Chemical Resources Laboratory, Tokyo Institute of Technology, Midori-ku, Yokohama, Japan, ${ }^{5}$ ICORP, ATP Synthesis Regulation Project, Japan Science and Technology Agency, National Museum of Emerging Science and Innovation, Koto-ku, Tokyo, Japan, ${ }^{6}$ Department of Cell Biology, Faculty of Medicine, Kyoto University, Yoshidakonoe-cho, Sakyo-ku, Kyoto, Japan and ${ }^{7}$ Human Receptor Crystallography Project, Exploratory Research for Advanced Technology (ERATO), Japan Science and Technology Agency, Yoshidakonoe-cho, Sakyo-ku, Kyoto, Japan
\end{abstract}

Vacuolar-type ATPases (V-ATPases) exist in various cellular membranes of many organisms to regulate physiological processes by controlling the acidic environment. Here, we have determined the crystal structure of the $A_{3} B_{3}$ subcomplex of V-ATPase at $2.8 \AA$ resolution. The overall construction of the $A_{3} B_{3}$ subcomplex is significantly different from that of the $\alpha_{3} \beta_{3}$ sub-domain in $F_{0} F_{1}$-ATP synthase, because of the presence of a protruding 'bulge' domain feature in the catalytic $A$ subunits. The $A_{3} B_{3}$ subcomplex structure provides the first molecular insight at the catalytic and non-catalytic interfaces, which was not possible in the structures of the separate subunits alone. Specifically, in the non-catalytic interface, the B subunit seems to be incapable of binding ATP, which is a marked difference from the situation indicated by the structure of the $F_{0} F_{1}$-ATP synthase. In the catalytic interface, our mutational analysis, on the basis of the $A_{3} B_{3}$ structure, has highlighted the presence of a cluster composed of key hydrophobic residues, which are essential for ATP hydrolysis by V-ATPases.

The EMBO Journal (2009) 28, 3771-3779. doi:10.1038/

emboj.2009.310; Published online 5 November 2009

*Corresponding authors. S Iwata, Division of Molecular Biosciences, Imperial College London, South Kensington Campus, London SW7 2AZ, UK. Tel.: + 44 (0)20 7594 3064; Fax: + 44 (0)20 7594 3065;

E-mail: s.iwata@imperial.ac.uk or K Yokoyama, Chemical Resource Laboratory, Tokyo Institute of Technology, Nagatsuda 4259, Midori-ku, Yokohama 226-8503, Japan. Tel.: + 8145924 5267; Fax: + 8145924 5277; E-mail: yokoyama.k.ab@m.titech.ac.jp

${ }^{8}$ These authors contributed equally to this work

${ }^{9}$ Present address: Centenary Institute, Locked Bag No. 6, Newtown,

New South Wales 2042, Australia

Received: 24 June 2009; accepted: 1 October 2009; published online: 5 November 2009
Subject Categories: proteins; structural biology Keywords: crystal structure; $\mathrm{F}_{\mathrm{o}} \mathrm{F}_{1}$; proton pump; rotary motor; V-ATPase

\section{Introduction}

The vacuolar-type ATPases/synthases (V-ATPases) are widely distributed in many organisms and are involved in a variety of physiological processes (Forgac, 2007). The eukaryotic $\mathrm{V}$-ATPases function as proton pumps that are involved in the acidification of cellular compartments such as the Golgi apparatus or lysosomes. In addition, some prokaryotes, such as the thermophilic eubacterium, Thermus thermophilus, contain a member of the family (or homologue) of the V-ATPases in their membranes (Yokoyama et al, 1990; Yokoyama and Imamura, 2005). The prokaryotic V-ATPase is made up of a simpler subunit composition than the eukaryotic enzymes, but each subunit shows significant sequence similarity to its eukaryotic counterpart (Tsutsumi et al, 1991; Yokoyama et al, 2003). For instance, the A subunit of T. thermophilus shows $49 \%$ sequence identity and $67 \%$ similarity to its human counterpart (Supplementary Figure S1A).

The V-ATPases and $\mathrm{F}_{\mathrm{o}} \mathrm{F}_{1}$-ATPases $\left(\mathrm{F}_{\mathrm{o}} \mathrm{F}_{1}\right)$, which function as ATP synthases in mitochondoria, chloroplast and most bacteria, are evolutionarily related and use a similar rotary catalytic mechanism that couples ATP hydrolysis/synthesis in the water soluble part of $F_{1}$ or $V_{1}$ with proton translocation across the membranes in the hydrophobic domain of $F_{o}$ or $V_{o}$ (Yoshida et al, 2001; Forgac, 2007). The isolated Escherichia coli $\mathrm{F}_{1}$, which has the subunit stoichiometry $\alpha_{3} \beta_{3} \gamma_{1} \delta_{1} \varepsilon_{1}$, hydrolyses ATP with high cooperativity between the three catalytic sites contained in the $\beta$ subunits at the $\alpha / \beta$ interfaces. The crystal structure of bovine $F_{1}$ revealed remarkable asymmetry in the conformation and nucleotide occupancy at the $\alpha-\beta$ interfaces, consistent with the binding change model for rotary catalysis (Boyer, 1993; Abrahams et al, 1994). In the binding change model, a central $\gamma$ subunit is surrounded by an $\alpha_{3} \beta_{3}$ cylinder, with the $\beta$ subunits at three catalytic interfaces bearing an ATP or ADP analogue. In this structure, two of the $\beta$ subunits $\left(\beta_{\mathrm{D}}, \beta_{\mathrm{T}}\right)$ at catalytic interfaces bearing nucleotides are in closed conformations, and the third $\beta$ subunit, which is free of bound nucleotides $\left(\beta_{\mathrm{E}}\right)$, has an open conformation. Sequential conformation change of each $\beta$ subunit (closed to open) during turnover is coupled to rotation of the $\gamma$ subunit. In reverse, rotation of the $\gamma$ subunit, which is driven by the proton motive force, drives sequential conformational changes in the $\beta$ subunits, resulting in ADP phosphorylation and release of product ATP from the enzyme.

Analysis of single particles by electron microscopy has indicated that the overall features of $\mathrm{V}_{1}$ are nearly identical to 
those of $F_{1}$. The A and B subunits also share $\sim 25 \%$ aminoacid sequence identity with the $\beta$ and $\alpha$ subunits of $F_{1}$, respectively. In particular, the ATP-binding regions of the catalytic $A$ subunits in $V_{1}$ and the $\beta$ subunits in $F_{1}$ are highly conserved. However, sequence alignment of the A subunit with the $\beta$ subunit revealed that an additional non-homologus region is present in the A subunit of $V_{1}$ (Forgac, 2007). Earlier mutagenesis studies have suggested that the nonhomologous region likely has an important function in energy coupling between the $V_{1}$ and $V_{0}$ moieties (Shao et al, 2003).

The $V_{1}$ of $T$. thermophilus is composed of an $A_{3} B_{3}$ cylinder and the D and $\mathrm{F}$ subunits (Yokoyama and Imamura, 2005). Experimental evidence for rotation in $V_{1}$ has been obtained by direct visualization of polystyrene beads attached to the $\mathrm{D}$ or $\mathrm{F}$ subunit, indicating that $\mathrm{V}_{1}$ catalyses ATP hydrolysis using a binding change mechanism similar to that of $\mathrm{F}_{1}$ (Imamura et al, 2003, 2005). On the basis of analogy with $F_{1}$ and several mutagenesis studies on V-ATPases, it has been proposed that ATP hydrolysis occurs at three catalytic sites sequentially, each located at the 'B-A interface', one of two interfaces between the A and B subunits (Yokoyama and Imamura, 2005; Forgac, 2007). Most residues in this site, including a P-loop motif responsible for nucleotide binding, are from the A subunit. The other interface, between the A and B subunits, is called the 'A-B interface'. The P-loop motif is not conserved in the $\mathrm{B}$ subunit and the isolated B subunit of $T$. thermophilus V-ATPase did not bind nucleotides, unlike the isolated A subunit (Yokoyama et al, 1998; Imamura et al, 2006). A reactive ATP analogue was, however, shown to modify the $\mathrm{B}$ subunit, which has been interpreted as possible nucleotide binding to the A-B interface (Manolson et al, 1985; Vasilyeva and Forgac, 1996).

Here, we present the X-ray structure of the V-ATPase $A_{3} B_{3}$ complex from T. thermophilus. The overall structure shows clear differences from that of the $\alpha_{3} \beta_{3}$ sub-domain in $F_{1}$ because of the presence of a protruding 'bulge' domain, belonging to the A subunit. Comparisons with the $F_{1}$ structure, in addition to mutagenesis studies at the $\mathrm{B}-\mathrm{A}$ and $\mathrm{A}-\mathrm{B}$ interfaces, have highlighted the importance of some hydrophobic residues in catalysis. We have also re-examined the reported nucleotide binding to the B subunits (supported by chemical modification), using a reactive ATP analogue. Our $\mathrm{X}$-ray structure also reveals many similarities and differences between the $F_{1}$ and $V_{1}$ at the catalytic interface, which contribute to a further understanding of the rotary catalytic mechanism of theses ATPases.

\section{Results and discussion}

\section{Structure determination of the $A_{3} B_{3}$ subcomplex}

The $A_{3} B_{3}$ complex, which does not contain nucleotides, was prepared as described earlier. The mutant $A_{3} B_{3}$ complex, in which all cysteine residues (A/C28, C255, C508, B/C265) were substituted with serine residues, was used for crystallization. The structure of $\mathrm{A}_{3} \mathrm{~B}_{3}$ was solved by molecular replacement, using partial structures of the A and B subunits from archea (PDB codes $1 \mathrm{VDZ}$ and 2C61, respectively) as search models. The crystallographic asymmetric unit contains two $A B$ dimers, which are virtually structurally identical. Extensive remodelling was required for both the A and B
Table I Summary of data collection and refinement

\begin{tabular}{|c|c|}
\hline & Native \\
\hline \multicolumn{2}{|l|}{ Data collection statistics } \\
\hline Resolution $(\AA)$ & $20-2.8(2.9-2.8)^{\mathrm{a}}$ \\
\hline Wavelength $(\AA)$ & 0.933 \\
\hline \multicolumn{2}{|l|}{ Number of reflections } \\
\hline Overall & 251463 \\
\hline Unique & $98563(9568)^{a}$ \\
\hline Completeness (\%) & $97.7(96.1)^{a}$ \\
\hline$I / \sigma$ & $11.6(2.02)^{\mathrm{a}}$ \\
\hline$R_{\text {merge }}(\%)^{\mathrm{b}}$ & $8.7(43)^{\mathrm{a}}$ \\
\hline \multicolumn{2}{|l|}{ Refinement statistics } \\
\hline Residue range & $\begin{array}{l}\text { A1-A578, B5-B464, } \\
\text { C1-C578, D5-D464 }\end{array}$ \\
\hline Number of non-hydrogen atoms & 16863 \\
\hline Number of water molecules & 707 \\
\hline Average temperature factor $\left(\AA^{2}\right)$ & 30.85 \\
\hline Resolution range $(\AA)$ & $20-2.8(2.87-2.8)^{\mathrm{a}}$ \\
\hline Twin fractions & $(h, k, l) 0.85 /(-h,-k, l) 0.15$ \\
\hline \multirow[t]{2}{*}{$R_{\text {cryst }}(\%)^{\mathrm{c}}$} & $25.0(28.3)^{\mathrm{a}}$ \\
\hline & $\begin{array}{l}\text { Without de-twinning } 27.2 \\
(34.1)^{\mathrm{a}}\end{array}$ \\
\hline Number of reflections & $93409(4990)^{\mathrm{a}}$ \\
\hline \multirow{2}{*}{$R_{\text {free }}(\%)^{\mathrm{d}}$} & $28.1(33.5)^{\mathrm{a}}$ \\
\hline & $\begin{array}{l}\text { Without de-twinning } 32.5 \\
(39.1)^{\mathrm{a}}\end{array}$ \\
\hline Number of reflections & $6485(312)^{\mathrm{a}}$ \\
\hline $\begin{array}{l}\text { Estimated overall coordinate } \\
\text { error }(\AA)\end{array}$ & 0.122 \\
\hline
\end{tabular}

RMS deviations from ideal values

$\begin{array}{ll}\text { Bond length }(\AA) & 0.011 \\ \text { Bond angles }(\mathrm{deg}) & 1.407 \\ \text { Torsion angles }(\mathrm{deg}) & 6.478 \\ \text { Chiral centre restraints }\left(\AA^{3}\right) & 0.092 \\ \text { General planes }(\AA) & 0.006\end{array}$

Ramachandran plot (non-Gly, non-Pro residues)

Residues in favoured and allowed $\quad 99.8$

regions (\%)

Residues in disallowed

0.1

regions (\%)

${ }^{a}$ Values for the highest resolution shell are given in parentheses.

${ }^{\mathrm{b}} R_{\text {merge }}=\sum_{h} \sum_{i}\left|I_{i}(h)-\langle I(h)\rangle\right| / \sum_{h} \sum_{i}(h)$, where $I_{i}(h)$ is the $i$ th measurement.

${ }^{\mathrm{c}} R_{\text {cryst }}=\sum\left\|F_{\mathrm{o}}|-| F_{\mathrm{c}}\right\| / \sum\left|F_{\mathrm{o}}\right|$, where $F_{\mathrm{o}}$ and $F_{\mathrm{c}}$ are observed and calculated structure factors, respectively.

${ }^{\mathrm{d}} R_{\text {free}}$ : The R-factor as defined above, but calculated using a subset $(5 \%)$ of reflections that are not used in the refinement.

subunit structures, because the isolated subunit structures were considerably different from those in the complex, particularly in the region of the subunit interfaces (Supplementary Figure S2). Accordingly, the $\mathrm{A}_{3} \mathrm{~B}_{3}$ complex structure provides much new information about the structures of the subunit interfaces, which are described below. The structure was refined using the data up to $2.8 \AA$ resolution. The data collection and refinement statistics are summarized in Table I. A small fraction of the crystal (15\%) is twinned with a twinning operator of $(-h,-k, l)$ and this contribution was included during the final refinement of the model (Supplementary Figure S3). The average B-factors for the $\mathrm{N}$-terminal, the bulge, the central $\alpha / \beta$ and the C-terminal domains of the A subunit are 18.0/18.0, 22.5/22.4, 29.6/29.6 and $46.3 / 46.3 \AA^{2}$, respectively (two numbers represent the values for two different $A$ subunits in the asymmetric unit). The average B-factors for the $\mathrm{N}$-terminal, the central $\alpha / \beta$ and 
A

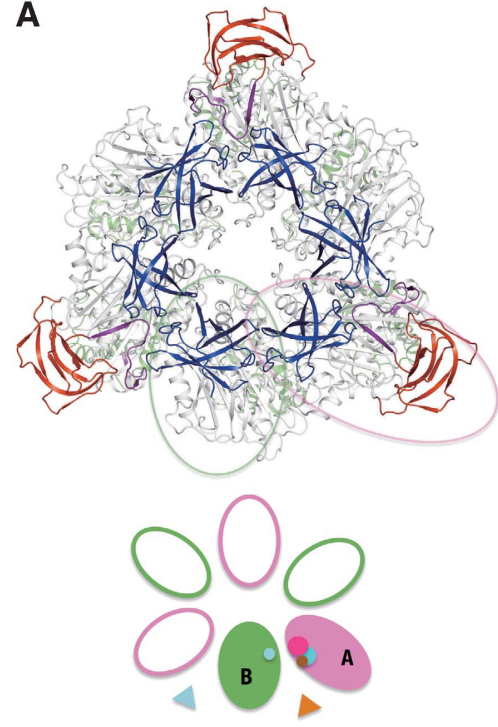

B

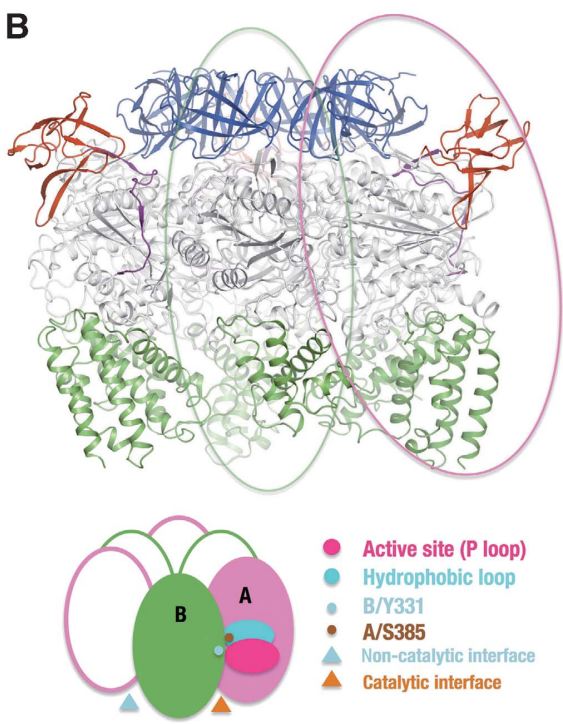

Figure 1 Crystal structure of the $A_{3} B_{3}$ complex. (A) Top view of the complex viewed towards the membrane. The $A$ and $B$ subunits are circled by magenta and green lines, respectively. (B) Side view of the $A_{3} B_{3}$ complex orthogonal to the three-fold axis. The $N$-terminal $\beta$-barrel domains, the $\alpha / \beta$ domains and the $\mathrm{C}$-terminal domains from the A and B subunits are shown in blue, light grey and green, respectively. The bulge domain and the extended linker from the A subunits are shown in red and purple, respectively. The catalytic and non-catalytic interfaces, the P-loop, the hydrophobic loop and two important amino-acid residues in the $\mathrm{A}_{3} \mathrm{~B}_{3}$ complex are schematically represented in the lower panels of the figure.

the C-terminal domains of the B subunit are 20.2/20.2, $29.1 / 29.1$ and $46.5 / 46.5 \AA^{2}$, respectively. As indicated by the average B-factors, the $\mathrm{C}$-terminal domains of the both $\mathrm{A}$ and $\mathrm{B}$ subunits are rather disordered and it was not possible to model side chains accurately. Other details are described in Materials and methods.

\section{Overall structure}

Figure 1 shows the top (A) and side (B) views of the $A_{3} B_{3}$ complex crystal structure. The three catalytic A subunits and three catalytic $B$ subunits are arranged alternately as in $F_{1}$. Both A and B subunits are nucleotide free in the crystal structure. The six A and B subunits in the complex are related by an approximate six-fold symmetry, which is highlighted by the N-terminal $\beta$-barrel domains (shown as blue ribbons). This six-fold symmetry, however, breaks down because of protruding structures from the A subunits (shown as red ribbons), which makes the $A_{3} B_{3}$ complex a triangular shape in the top view. This protruding structure, referred to as the bulge hereafter, is encoded by the 'non-homologus region' of the $A$ subunit that is not conserved in $F_{1}$. In the side view, perpendicular to the molecular three-fold axis, the $A_{3} B_{3}$ complex structure does not have the orange-like spherical shape of the $F_{1}$ (Abrahams et al, 1994). The main structural differences between the V-ATPase $A_{3} B_{3}$ complex and the $\alpha_{3} \beta_{3}$ sub-domain in $F_{1}$ are observed in two regions in the $A$ subunit. One is the bulge mentioned above and the other is the presence of three longer helices in the C-terminal domain facing the $V_{o}$ domain in the membrane. The $A_{3} B_{3}$ complex maintains a large internal cavity, which should accommodate a part of the DF central shaft in the full complex. The diameter of the cavity is larger than those observed for the $\alpha_{3} \beta_{3}$ sub-domain in $F_{1}$ because all A subunits in the complex are present in an 'open' conformation as described below.
$B$ subunit structure and the non-catalytic $A-B$ interface The $B$ subunit in the $A_{3} B_{3}$ complex consists of the $\mathrm{N}$-terminal $\beta$-barrel domain (5-79), the central $\alpha / \beta$ domain (80-373) and the C-terminal domain (374-464), as shown in Figure 2. Secondary structure elements of A and B subunits are summarized in Supplementary Figure S4. The B subunit has been suggested to be the non-catalytic subunit, equivalent to the $\mathrm{F}_{1}-\alpha$ subunit (Manolson et al, 1985; Vasilyeva and Forgac, 1996; Forgac, 2007). This was examined by comparing the $B$ subunit structure with $\alpha$ and $\beta$ subunit structures of yeast $F_{1}$, respectively (Kabaleeswaran et al, 2006; PDB ID: 2HLD). In the $F_{1}$ structure, all three $\alpha$ subunits adopt similar conformations, whereas the $\beta$ subunits are in three distinct conformations, namely $\beta_{\mathrm{D}}, \beta_{\mathrm{T}}$ and $\beta_{\mathrm{E}}$ (Abrahams et al, 1994). Using the $C^{\alpha}$ atom positions, the whole $B$ subunit can be superimposed onto the best fit $\alpha$ and $\beta\left(\beta_{\mathrm{E}}\right)$ subunits with r.m.s.d. values of 1.7 and $1.9 \AA$, respectively, when the molecule 2 of 2 HLD is used. This means that the nucleotidefree $B$ subunit structure is more similar to the $\alpha$ subunit structure with bound AMPPMP than to the nucleotide-free $\beta$ subunit $\left(\beta_{\mathrm{E}}\right)$ structure. The similarity of these two subunits is highlighted when their domains are compared. The N-terminal $\beta$-barrel, central $\alpha / \beta$ and $\mathrm{C}$-terminal domains of the $\mathrm{B}$ subunit can be superimposed onto the respective domains of the $\alpha$ subunit, with r.m.s.ds of $1.7,1.3$ and $1.6 \AA$, respectively.

It has been thought that the $\mathrm{B}$ subunit could contain a nucleotide-binding site because it is modified by the ATP analogue of 3-O-(4-benzoyl) benzoyladenosine $5^{\prime}$-triphosphate (Bz-ATPase) or 2-azido-ATP (Manolson et al, 1985). On the other hand, the structure of the potential nucleotidebinding site of the $\mathrm{B}$ subunit in the $\mathrm{A}-\mathrm{B}$ interface is very different from that observed in the $\beta$ - $\alpha$ interface of $F_{1}$, which binds a non-catalytic nucleotide. Unlike the non-catalytic $\alpha$ subunit, the P-loop sequence (GXXXXGKT/S, also known as 'Walker motif $A^{\prime}$ ) is not conserved in the B subunit. 

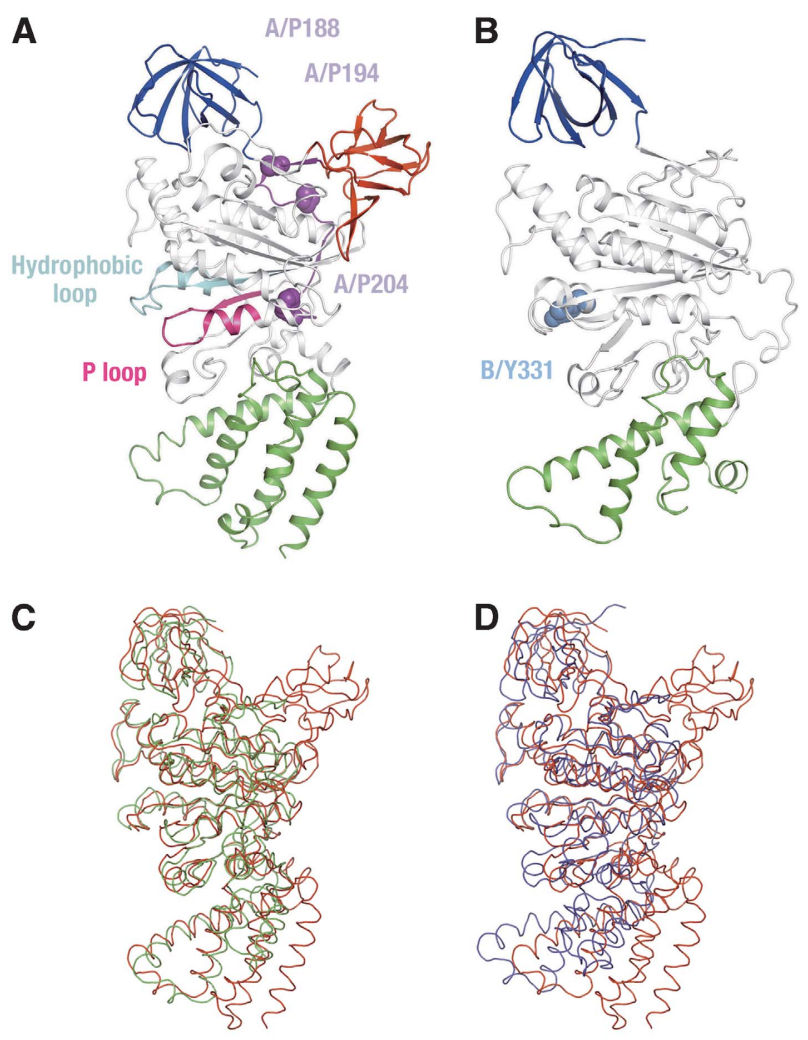

A versus $\beta_{\mathrm{E}}$

Figure 2 Structures of the A and B subunits in the complex. (A) A subunit structure. The $\beta$-barrel (A1-72) and the C-terminal (A442$565)$ domains are shown in blue and green, respectively. The bulge domain (A113-184), the extended linker (A185-204), the P-loop (A222-250) and the hydrophobic loop (A374-407) are highlighted in red, purple, magenta and cyan, respectively. The conserved proline residues in the extended linker are shown in purple and labelled. These proline residues have been reported to be important for the coupling efficiency of yeast V-ATPase (Shao et al, 2003). (B) B subunit structure. The $\beta$-barrel (B5-79) and the C-terminal (B374-464) domains are shown in blue and green, respectively. Tyrosine 331 in the B subunit is shown in sky blue. (C) A subunit (red) superimposed on the $\beta_{E}$ subunit of the yeast $F_{1}$. (D) A subunit (red) superimposed on the $\beta_{\mathrm{D}}$ (blue) subunit of the yeast $\mathrm{F}_{1}$.

Figure 3 compares the structures of the P-loop equivalent region in the $\mathrm{B}$ subunit and of the nucleotide-bound P-loop in the yeast $F_{1} \alpha_{E}$ subunit. The P-loop has a unique main-chain structure, which allows five amide protons within the loop to form hydrogen-bonding interactions (shown in blue in Figure 3B) with the triphosphate group of bound ATP. The equivalent loop in the $B$ subunit shows a totally different main-chain conformation, which by analogy does not allow the formation of these hydrogen bonds (Figure 3A and C). This is presumably because the P-loop equivalent region in the B subunit lacks flexible glycine residues and includes an insertion of a proline residue, which does not have an amide group. In addition, several residues known to be involved in nucleotide binding at the $\beta-\alpha$ interface $(\beta / \mathrm{Y} 368, \beta / \mathrm{R} 372$, $\alpha / \mathrm{Q} 172, \alpha / \mathrm{Q} 432, \alpha / \mathrm{P} 367$, the bovine $\mathrm{F}_{1}$ residue numbering is used hereafter) are missing in the $A_{3} B_{3}$ complex. In the $F_{1}$ structure, the side chains of $\alpha / \mathrm{Q} 172, \alpha / \mathrm{K} 175, \alpha / \mathrm{T} 176$ in the P-loop region interact with the diphosphate group of ATP-Mg at the $\beta_{\mathrm{T}}-\alpha_{\mathrm{E}}$ interface. Substitution of these residues with

alanine causes the complete loss of ATP binding to the $\alpha$ subunit, resulting in a dramatic loss of steady-state ATP hydrolytic activity (Matsui et al, 1997). In contrast, a double mutant of $\mathrm{V}_{1}$ (B/N161/A (equivalent to $\left.\alpha / \mathrm{T} 176\right)$ and B/E162A (equivalent to $\alpha / A 177)$ ), which eliminates hydrophilic residues on the loop region, shows a comparable ATP-hydrolysis rate to that of the wild-type $\mathrm{V}_{1}$ (Table II). These mutations did not affect the profile of ATP hydrolysis of $V_{1}$ either (Supplementary Figure S6). In conclusion, it is unlikely that the $\mathrm{B}$ subunit binds a nucleotide at the $\mathrm{A}-\mathrm{B}$ interface in a similar manner to that of the $\alpha$ subunit. The chemical modification of the $\mathrm{B}$ subunit by nucleotide analogues can be explained as a modification of the $B$ subunit residues in the catalytic B-A interface, but not in the A-B interface. As discussed below, we found a conserved B/Y331 at the nucleotide-binding site in the B-A interface, which is the likely target of this modification.

\section{A subunit structure}

The structure of the A subunit is shown in Figure 2A. The A subunit consists of four domains: the $\mathrm{N}$-terminal $\beta$-barrel domain (1-72), the non-homologus domain including the bulge (113-184), the central $\alpha / \beta$ domain (205-441) and the C-terminal domain (442-565). The A subunit is the catalytic subunit of the V-ATPases and is equivalent to the $F_{1}-\beta$ subunit. As shown in Figure 2C and Table II, structures of the $A$ and the $\beta$ subunits are similar except for the presence of an additional bulge of eight $\beta$-strands and a larger $\mathrm{C}$-terminal $\alpha$-helical bundle in the A subunit. The bulge is connected to the nucleotide-binding domain through an extended linker (A185-204), which starts in the space between the N-terminal and the bulge domains and runs down the surface of the $\mathrm{A}_{3} \mathrm{~B}_{3}$ complex (Figure 2A; Supplementary Figure S4A). Both the bulge and this extended linker are encoded by the socalled 'non-homologus' region of sequence (Shao et al, 2003). Interestingly, the residues within this linker (A/P188, A/P194 and A/P204) are conserved among V-ATPases (Supplementary Figure S1A). Substitution of the equivalent residues in yeast $\mathrm{V}$-ATPase results in a change in coupling efficiency (Shao et al, 2003). These results suggest that the linker region is involved in the interaction between the peripheral stalk (composed of the $\mathrm{E}$ and $\mathrm{G}$ subunits) and the $A_{3} B_{3}$ complex.

The presence of longer helices, including $\alpha 13-\alpha 16$ in the $\mathrm{C}$-terminal domain, distinguishes the domain structure from that of the equivalent $F_{1}-\beta$ subunit (Figure 2D; Supplementary Figure S3A). This structure is also conserved in the isolated A subunit of Pyrococcus horikoshii (Maegawa et al, 2006), indicating that this is a general feature observed among V-ATPase structures.

The A subunit superimposes well onto the structure of the $F_{1}-\beta_{\mathrm{E}}$ subunit, but not onto those of the $\beta_{\mathrm{D}}$ and $\beta_{\mathrm{T}}$ subunits (Figure $2 \mathrm{C}$ and $\mathrm{D}$ ). This is because of conformational differences around the nucleotide-binding site of nucleotide-free $\left(\beta_{\mathrm{E}}\right)$ and nucleotide-bound $\beta$ subunits (Menz et al, 2001). A similar conformational change should be induced in the A subunit by nucleotide binding and/or interaction with the central rotor. That is, we predict that the lower part of the nucleotide-binding domain (205-245, 405-441) will rotate upwards, together with the C-terminal domain (442-565), as one group. This conformational change should act to place side chains critical for catalysis into the correct positions and 


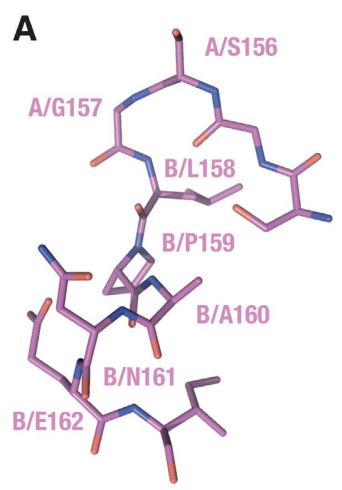

V-ATPase

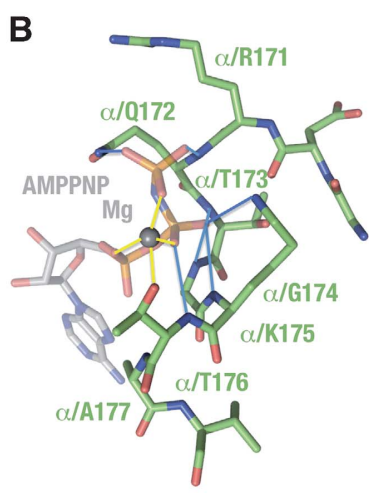

F-ATPase

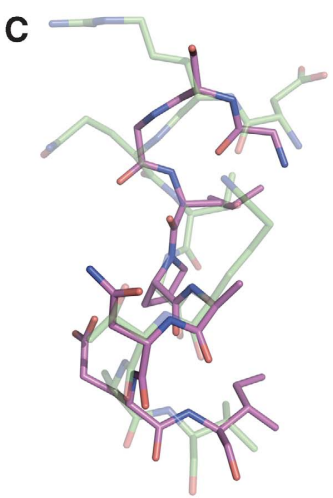

Figure 3 Structures of the P-loop equivalent region in the B subunit and of the nucleotide-bound P-loop in the yeast $\mathrm{F}_{1} \alpha_{\mathrm{E}}$ subunit. (A) Structure of the P-loop equivalent region in the B subunit. (B) Structure of the P-loop of the yeast $F_{1} \alpha$ subunit. The bound AMP-PNP molecule and the magnesium ion are shown. Hydrogen bonds are shown in light blue, whereas coordination links to the magnesium ion are shown in yellow. (C) Superposition of (A) and (B). Only protein atoms are shown.

Table II Effect of mutagenesis on the ATP hydrolysis activity of $\mathrm{V}_{1} / \mathrm{F}_{1}$

\begin{tabular}{|c|c|c|c|c|c|}
\hline \multicolumn{3}{|c|}{$\mathrm{V}_{1}$} & \multicolumn{3}{|c|}{$\mathrm{F}_{1}$} \\
\hline Mutation & Turnover rate $\left(\mathrm{s}^{-1}\right)$ & Relative activity (\%) & Mutation & Turnover rate $\left(\mathrm{s}^{-1}\right)$ & Relative activity (\%) \\
\hline Wt & $108 \pm 13$ & 100 & $\mathrm{Wt}$ & $321 \pm 13$ & 100 \\
\hline A/E257D & $4.26 \pm 0.29$ & 3.9 & $\beta / Y 311 F$ & $170 \pm 3.6$ & 53.0 \\
\hline A/E257Q & $0.19 \pm 0.04$ & $<1.0$ & $\beta / Y 311 S$ & $1.2 \pm 0.5$ & $<1.0$ \\
\hline A/Y419Q & $0.37 \pm 0.03$ & $<1.0$ & $\beta / \mathrm{Y} 311 \mathrm{~A}$ & $2.2 \pm 0.4$ & $<1.0$ \\
\hline $\mathrm{B} / \mathrm{R} 360 \mathrm{~K}$ & $0.14 \pm 0.02$ & $<1.0$ & & & \\
\hline $\mathrm{B} / \mathrm{R} 360 \mathrm{~L}$ & $0.13 \pm 0.02$ & $<1.0$ & & & \\
\hline B/Y331S & $0.09 \pm 0.02$ & $<1.0$ & & & \\
\hline $\mathrm{B} / \mathrm{Y} 331 \mathrm{~F}$ & $48.1 \pm 10.0$ & 44.3 & & & \\
\hline B/Y331L & $0.49 \pm 0.08$ & $<1.0$ & & & \\
\hline $\mathrm{B} / \mathrm{Y} 331 \mathrm{~A}$ & $0.03 \pm 0.01$ & $<1.0$ & & & \\
\hline $\mathrm{A} / \mathrm{F} 230 \mathrm{~A}$ & $6.00 \pm 3.22$ & 5.5 & & & \\
\hline $\mathrm{A} / \mathrm{S} 385 \mathrm{~A}$ & $1.24 \pm 0.19$ & 1.1 & & & \\
\hline B/N161A-E162A & $116.6 \pm 1.4$ & 107 & & & \\
\hline
\end{tabular}

The ATP hydrolysis activities of each mutant enzyme were measured by the enzyme-coupling assay. Each value represents the average of 3-5 replicates. The $\mathrm{V}_{1}$ contains the TSSA mutation as described in Materials and methods. Note that the residue numbers of bacillus PS3 $\mathrm{F}_{1}$ are on the basis of the bovine $\mathrm{F}_{1}$ system.

conformations for nucleotide binding, as we discuss in the following section.

Regulatory disulphide bond formation between conserved cysteine residues in the A subunit (A/C261 and C539 in yeast V-ATPase) has been proposed (Feng and Forgac, 1994). The equivalent cysteine residues in the T. thermophilus A subunit are A/S232 and A/S539 (mutated from A/C539), respectively. In our open structure of $A$ subunit, the distance between the hydroxyl oxygen atom of A/S232 and the sulfur atom of A/C507 is $26 \AA$ apart. It is, therefore, unlikely that a disulphide bridge exists between them. However, it is possible that side chain of A/S232 might be proximate to that of A/C507 when the A subunit adopts the closed conformation. Disulphide bond formation between two different subunits is unlikely because the relevant residues are more than $60 \AA$ apart.

\section{The catalytic B-A interface}

The nucleotide-binding sites are located in the interface between the $\mathrm{B}$ and $\mathrm{A}$ subunits. The site is mainly composed of the residues from the A subunit, but some from the $\mathrm{B}$ subunit are also essential. The nucleotide-binding domain of the A subunit shows high sequence similarity to that of $F_{1}$, including the P-loop, which is responsible for coordination of phosphate moieties of ATP (Supplementary Figure S1A). Figure 4 shows a structural comparison between the nucleotide-binding sites of the $\mathrm{V}_{1} \mathrm{~B}-\mathrm{A}$ interface and the $\alpha_{E}-\beta_{E}$ interface in the yeast $F_{1}$. As expected, the binding site in the B-A interface is similar to that in the $\alpha_{\mathrm{E}}-\beta_{\mathrm{E}}$ interface in the $F_{1}$, including the P-loop. For example, the $A$ subunit structure confirmed that residues A/K234 and A/ T235 are placed in similar positions to those of the $\beta / \mathrm{K} 162$ and $\beta /$ T163 residues of the $\beta$ subunit, respectively. The superposition of both interfaces, however, identified some significant differences (Figure 4). Electron density maps calculated during the determination of the yeast $F_{1}$ structure allowed identification of density attributed to bound inorganic phosphate $\left(\mathrm{P}_{i}\right)$. However, equivalent analysis indicates that the $\mathrm{B}-\mathrm{A}$ interface in the present structure is $\mathrm{P}_{i}$ free (Figure 4A). In the yeast $\mathrm{F}_{1}$ structure, the bound $\mathrm{P}_{i}$ was coordinated by residues $\beta / \mathrm{K} 162$ (equivalent to $A / K 234$ ), $\beta /$ R189 (A/R258), $\beta / \mathrm{D} 256$ (A/D325), $\beta / R 260$ (A/R329), $\alpha /$ R373 (B/R360) and $\beta / \mathrm{N} 257$ (A/S326). In contrast, the A-subunit structure shows that all arginine residues are orientated away from the putative $\mathrm{P}_{i}$-binding site, leaving the site much more open and structurally less favourable for $\mathrm{P}_{i}$ binding (Figure $4 \mathrm{~B}$ ). 

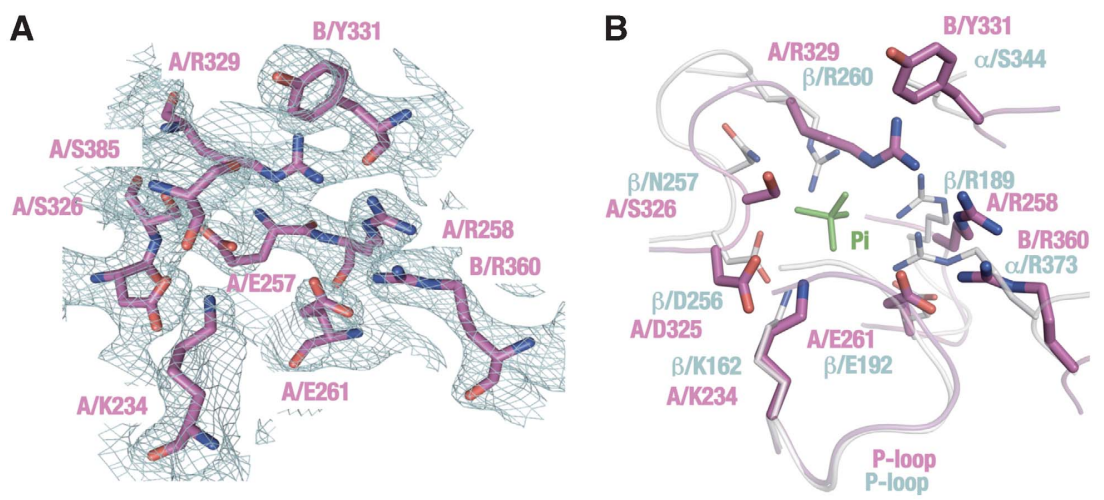

Figure 4 Structure of the B-A catalytic interface. (A) Electron density map of the B-A catalytic interface. Contours are drawn at $1.2 \sigma$. Aminoacid side chains essential for catalysis are labelled. (B) Superposition of the B-A interface (magenta) and the $\alpha_{\mathrm{E}}-\beta_{\mathrm{E}}$ interface of the yeast $\mathrm{F}_{1}$ (light blue). A bound phosphate molecule at the $\alpha_{\mathrm{E}}-\beta_{\mathrm{E}}$ interface is shown in green. Note that the residue numbers are on the basis of the bovine $\mathrm{F}_{1}$ system.

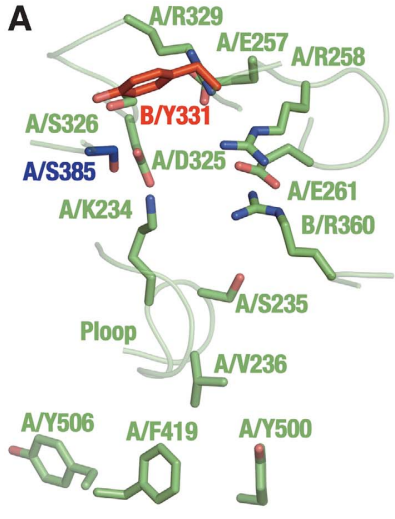

V-ATPase (open form)

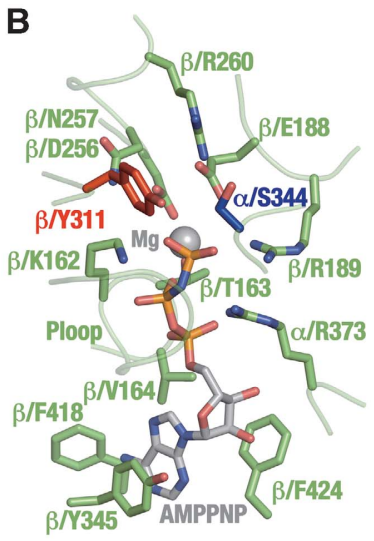

F-ATPase (closed form)

Figure 5 Comparison of the B-A interface of V-ATPase and the $\alpha_{D}-\beta_{D}$ interface of the yeast $F_{1}$. (A) Side chains essential for catalysis in the B-A interface. A/S385 and B/Y331 are highlighted in blue and red, respectively. (B) Side chains essential for catalysis in the $\alpha_{D}-\beta_{D}$ interface of the yeast $F_{1} . \beta / Y 311$ and $\alpha / S 344$ are highlighted in grey, red and blue, respectively. The bound AMPPNP ligand and the magnesium ion are shown. Note that the residue numbers are on the basis of the bovine $F_{1}$ system.

We have also compared the $\mathrm{B}-\mathrm{A}$ interface structures of $V$-ATPase with the nucleotide-bound $\alpha_{D}-\beta_{D}$ interface of the yeast $F_{1}$. Figure 5 shows that most of the key residues for nucleotide binding and catalysis are conserved, except for $\alpha / \mathrm{S} 344$ (B/Y331), which will be discussed below. As our V-ATPase structure is nucleotide free, we have performed some mutational studies to confirm that this is the catalytic site of the $V_{1}$. The subunit stoichiometry of mutated $F_{1} / V_{1}$ has been confirmed by SDS-PAGE (Supplementary Figure S5). $\mathrm{A} / \mathrm{E} 257$ is well conserved in the A subunits among species and is thought to be equivalent to residue $\beta / E 188$ (Supplementary Figure $\mathrm{S} 1 \mathrm{~A}$ ). In $\mathrm{F}_{1}$, the side chain of $\beta / \mathrm{E} 188$ is appropriately positioned close to the nucleophilic water molecule at the $\alpha_{D}-\beta_{D}$ interface, promoting an inline nucleophilic attack on the terminal $\gamma$-phosphate moiety of the substrate ATP. The mutation of A/E257 in $V_{1}$ to aspartate or glutamine resulted in almost complete loss of ATP-hydrolysis activity (Table II), consistent with earlier results with the yeast V-ATPase (Liu and Kane, 1996). The guanidinium moiety of $\alpha /$ R373 at the $\alpha-\beta$ interface in the $F_{1}$ has a critical

function in catalysis by stabilizing the negative charge of the $\gamma$-phosphate group in the transition state. The mutation of $B / R 360$, equivalent to $\alpha / R 373$, to lysine or leucine causes complete loss of ATP-hydrolysis activity in $\mathrm{V}_{1}$ (Table II), as does the equivalent mutation of $\alpha /$ R373 in $F_{1}$. Residues in the adenine-binding pocket, as identified in the $F_{1} \beta$ structure, are well conserved in the subunit A structure from $V_{1}$. Accordingly, the mutation of $A / F 419$, which is equivalent to $\beta / Y 345$, to glutamate causes a complete loss of ATP-hydrolysis activity (Table II). Taken together, these mutation results confirm that the catalytic sites of $F_{1}$ and $V_{1}$ are closely related.

We have, however, observed one interesting and important difference in the nucleotide-binding sites of two ATPases. The residue $\alpha / \mathrm{S} 344$ is well conserved in $\mathrm{F}_{1}$ among species, and in the elucidated structures, it lies in close proximity to the catalytic water interacting with $\beta / \mathrm{E} 188$ at the $\alpha_{D}-\beta_{D}$ interfaces (Abrahams et al, 1994). Thus, the $\alpha /$ Ser344 residue has been thought to be critical for catalysis (Abrahams et al, 1994; Menz et al, 2001; Dittrich et al, 2004). However, the serine residue is not conserved at the identical position in the $B$ subunit of $V_{1}$ (Supplementary Figure S1B). Instead, the conserved tyrosine residue $(\mathrm{B} / \mathrm{Y} 331)$ is located at the identical position. Mutation of residue B/Y331 to serine, alanine or leucine reduced the ATPase activity of $V_{1}$ by $>99 \%$ (Table II). In contrast, the mutant protein $\mathrm{B} / \mathrm{Y} 331 \mathrm{~F}$ showed comparable activity (ca $45 \%$ of residual activity) to that of the wild-type protein. These results are consistent with earlier mutational studies of the yeast V-ATPase (Liu and Kane, 1996). Therefore, an aromatic side chain in this position seems essential for the ATP-hydrolysis activity of $V_{1}$. Interestingly, we have also identified an aromatic side chain $(\beta / Y 311)$, which is located next to $\alpha / \mathrm{S} 344$, and which occupies the equivalent position in the $\alpha_{D}-\beta_{D}$ interface in the structure of $F_{1}$. This $\beta / Y 311$ residue can react with hydrophobic chemical modifers such as $\mathrm{NBf}-\mathrm{Cl}$ or 8 -azide ATP (Yoshida and Allison, 1990; Garin et al, 1994), even though the residue lies $\sim 7 \AA$ away from the adenine pocket (Abrahams et al, 1994). Moreover, the serine residue is conserved among $\mathrm{V}_{1}$ proteins (A/S385 in the Thermus $\mathrm{V}_{1}$ ) at the equivalent position to residue $\beta / \mathrm{Y} 311$ in $F_{1}$. It seems that the $\mathrm{B} / \mathrm{Y} 331-\mathrm{A} / \mathrm{S} 385$ pair in the $\mathrm{V}_{1}$ has the same function as the $\alpha / \mathrm{S} 344-\beta /$ Y311pair in the $F_{1}$ with swapped positions.

To prove this hypothesis, we designed several mutational studies. As expected, the A/S385A substitutions caused 
complete loss of ATPase activity of the $\mathrm{V}_{1}$ (Table II). Similar to $\alpha /$ S344 in the $F_{1}, A / S 385$ possibly coordinates a catalytic water molecule at the nucleotide-bound B-A interfaces. $\beta /$ Y311 of $F_{1}$ at the equivalent position to $A / S 385$ was also mutated using the $F_{1}$ from the thermophilic bacillus PS-3. Both $\beta /$ Y311S and $\beta /$ Y311A substitutions significantly decreased the ATP-hydrolysis activity of $F_{1}$, but the $\beta / Y 311 F$ substitution showed weak effect on the activity (Table II). In conclusion, the pair of a serine and an aromatic residue seems essential for catalysis in both $\mathrm{F}_{1}$ and $\mathrm{V}_{1}$.

\section{Importance of hydrophobic residues at the B-A interface} It is not immediately obvious how aromatic residues such as $\mathrm{B} / \mathrm{Y} 331$ and $\beta / \mathrm{Y} 311$ can be involved in ATP hydrolysis. Our $\mathrm{A}_{3} \mathrm{~B}_{3}$ structure enables us to compare the catalytic interfaces of both ATPases. By combining the structural information and mutational studies, we have studied the importance of these hydrophobic residues. Figure 6 compares the surroundings of these hydrophobic residues in the V-ATPase (A) and nucleotide free and bound $\mathrm{F}_{1}$ structures (open (B) and closed (C) conformations, respectively). In the $\mathrm{F}_{1}$, nucleotide binding induces a conformation change, which relocates the $\beta 132-173$ region, including the P-loop, towards the $\beta 330-$ 464 region, including the $\mathrm{C}$-terminal domain and its linker to the central $\alpha / \beta$ domain (Figure $6 \mathrm{~B}$ and $\mathrm{C}$ ). In this closed conformation, the side chains from both regions, including residues $\beta / \mathrm{F} 155, \beta / \mathrm{A} 158, \beta / \mathrm{I} 310, \beta / \mathrm{Y} 311, \beta / \mathrm{P} 313$ and $\beta / \mathrm{F} 326$ form a hydrophobic cluster at the interface (Figure $6 \mathrm{C}$ ). For example, $\beta / \mathrm{F} 155$ and $\beta / \mathrm{A} 158$, which are associated with the P-loop, move into contact with $\beta / F 326$ and $\beta / P 313$, respectively. This closure isolates the catalytic site, defined by these hydrophobic residues and facilitates the correct alignment of water molecules and residues essential for catalysis. Computer simulations show that ATP hydrolysis at the catalytic site of the $F_{1}$ requires a multi-centre proton pathway comprised of amino-acid side chains and several water molecules, including the nucleophilic water molecule coordinated with $\beta / \mathrm{E} 188$ and $\alpha / \mathrm{S} 344$ (Dittrich et al, 2004).

Although hydrophobic residues including A/F230, A/P386, $\mathrm{A} / \mathrm{P} 387, \mathrm{~A} / \mathrm{P} 220, \mathrm{~A} / \mathrm{L} 400$ and $\mathrm{B} / \mathrm{Y} 331$ are observed in the V-ATPase $A_{3} B_{3}$ structure at equivalent locations, the residue identities are not strictly conserved. For example, the aromatic side chains of $\beta / F 155$ and $\beta / F 326$, which show stacking orientations in the closed form of $F_{1}$, are replaced by
A/P227 and A/L400 in the V-ATPase. $\beta / A 158$ in the P-loop is replaced by $A / F 230$, which is highly conserved in the $\mathrm{V}$-ATPases. If the closed form of the $\mathrm{V}_{1}$ is similar to that observed for the $F_{1}$, then residue $A / F 230$ should move into proximity with $\mathrm{A} / \mathrm{P} 387$ and $\mathrm{B} / \mathrm{Y} 331$ to form a hydrophobic cluster, in which the residues interact very differently from those in the $F_{1}$. This idea is strongly supported by the result that the substitution of $A / F 230$ by alanine caused nearly complete loss of the ATPase activity of $\mathrm{V}_{1}$ (Table II). In conclusion, the formation of a hydrophobic cluster has a critical role in catalysis for both the $\mathrm{V}_{1}$ and $\mathrm{F}_{1}$ rotary motors. Observed differences in the cluster possibly affect the stability of the closed conformations of the $A$ and $\beta$ subunits, thus affecting the open-closed transition of these catalytic subunits during rotary catalysis.

Interestingly, similar hydrophobic cluster formation has been reported in the sarcoplasmic reticulam $\mathrm{Ca}^{2+}$-ATPase (Yamasaki et al, 2008), which is a P-type ion transporting ATPases that catalyses $\mathrm{Ca}^{2+}$ transport coupled with ATP hydrolysis. The crystal structures of the E1P $\mathrm{Ca}^{2+} 2$ and E2P states of the $\mathrm{Ca}^{2+}$-ATPase showed that a hydrophobic cluster is formed at the interface between the phosphorylation (P) and actuator (A) domains, during the transition between the two states (Olesen et al, 2007). The cluster is formed by seven residues, including Y122, which are located on the interface, and mutational studies on the residues in the cluster indicate that the formation of the hydrophobic cluster is critical for the catalytic cycle of the $\mathrm{Ca}^{2+}$-ATPase. Their proposal supports our idea that hydrophobic cluster formation is important for the ATPase reaction in rotary ATPases.

\section{Materials and methods}

\section{Purification, crystallization and structure determination}

The $A_{3} B_{3}$ subcomplex overexpressed in E. coli BL21-CodonPlus (DE3)-RP (Stratagene) was purified, as described earlier (Imamura et al, 2003). The purified $A_{3} B_{3}$ complex was supplemented with a final concentration of $100 \mathrm{mM} \mathrm{MgCl}$ and concentrated to a protein concentration of $10 \mathrm{mg} / \mathrm{ml}$ using a Centricon $100 \mathrm{k}$ concentrator (Millipore). Crystals were obtained by the vapour diffusion method in a hanging drop with the reservoir solution, $11.5 \%$ (w/v) PEG 8000, 0.1 M Tris- $\mathrm{HCl}$ (pH 7.5), 5\% (v/v) MPD and $100 \mathrm{mM} \mathrm{MgCl}_{2}$. By adding $20 \%$ glycerol, the reservoir solution was used to flash freeze the crystals in liquid nitrogen. X-ray data were collected at $100 \mathrm{~K}$ using an ADSC Quantum-4 CCD detector with a wavelength of $0.933 \AA$ and $0.5^{\circ}$ oscillation steps over a range of $120^{\circ}$ at the ESRF

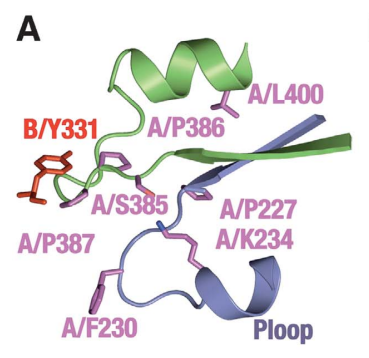

V-ATPase (open)

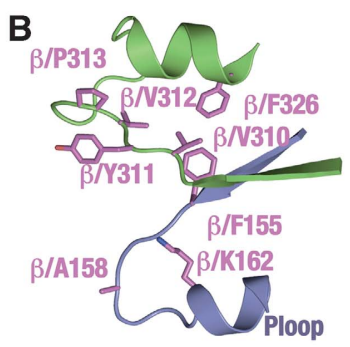

F-ATPase (open)

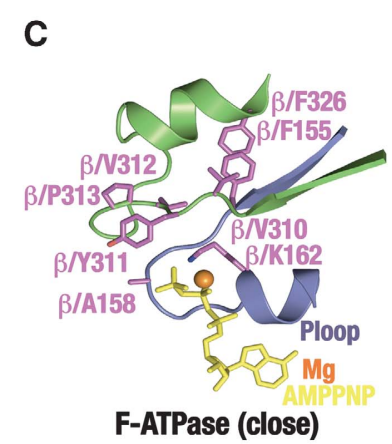

F-ATPase (close)

Figure 6 Hydrophobic cluster formation in the catalytic interfaces of V-and F-ATPases. (A) Schematic representation of the P-loop (A223-239, violet) and the hydrophobic loop (A379-403, green) in the B-A interface of V-ATPase. B/Y331 is shown in red. (B) Schematic representation of the P-loop ( $\beta 152-167)$ and the hydrophobic loop $(\beta 300-322)$ in the $\alpha_{E}-\beta_{E}$ interface of the yeast $F_{1}$. Note that the residue numbers of the yeast $F_{1}$ in this figure are on the basis of the bovine $\mathrm{F}_{1}$ system. (C) Schematic representation of the P-loop and the hydrophobic loop in the $\alpha_{\mathrm{E}}-\beta_{\mathrm{D}}$ interfaces of the yeast $\mathrm{F}_{1}$. The bound AMPPMP molecule and the magnesium ion are shown. 
ID14-2 beamline (Grenoble, France). The data set was processed to $2.8 \AA$ resolution using the programs Denzo and Scalepack (Otwinowski and Minor, 1997) and the CCP4 program suite (Collaborative Computational Project, Number 4, 1994). The crystal belonged to the space group P321, with unit cell dimensions $a=b=199.4 \AA$ and $c=179.0 \AA$. The average $\mathrm{B}$-factor estimated from the Wilson plot was $62.5 \AA^{2}$. The $2.8 \AA$ resolution structure of the $\mathrm{A}_{3} \mathrm{~B}_{3}$ subcomplex was solved by molecular replacement with PHASER (McCoy et al, 2007), using partial structures of the A and B subunits from archea (PDB code $1 \mathrm{VDZ}$ and 2C61) as search models (Maegawa et al, 2006; Schafer et al, 2006). A single solution with two $\mathrm{AB}$ heterodimers located close to the crystallographic three-fold axis was identified (Supplementary Figure S3A and B). The two dimers were related by a translational symmetry, which is confirmed by peaks at $(0.66,033,0.02)$ in the native Patterson map. Preliminary rigid body and restrained refinement with REFMAC5 (Murshudov et al, 1997) gave residuals $R=0.44$ and $R_{\text {free }}=0.48$ for this solution. Model bias was minimized by solvent flattening using RESOLVE (Terwilliger, 2000) using 'prime-and-switch' phasing and two-fold NCS averaging. The resulting electron density maps were readily interpretable and clearly showed areas with structural differences. Manual adjustments to the model were made using COOT (Emsley and Cowtan, 2004) and O (Jones et al, 1991). Model refinement was carried out using CNS (Brunger et al, 1998) and REFMAC5 with TLS (Painter and Merritt, 2006). We found that a small fraction of the crystal $(15 \%)$ is twinned with a twinning operator $(-h,-k, l)$ and this contribution was included in the final stage of the refinement using REFMAC5 (Supplementary Figure $\mathrm{S} 3 \mathrm{C})$. This improved the values of the residuals, $R$ and $R_{\text {free }}$ to $25.0 \%$ (from $27.3 \%$ ) and $28.1 \%$ (from $32.8 \%$ ), respectively.

\section{Biochemical assay}

The $\mathrm{V}_{1}$ of $T$. thermophilus and $\mathrm{F}_{1}$ of thermophilic Bacillus PS-3 were expressed in $E$. coli and purified by earlier described procedures
(Imamura et al, 2003). We used the TSSA mutant $\mathrm{V}_{1}\left(\mathrm{~A}_{(\mathrm{His}-10 / \mathrm{s} 232 \mathrm{~A} /}\right.$ ${ }_{\mathrm{T} 235 \mathrm{~S}) 3} \mathrm{~B}_{3} \mathrm{DF}$ ) to avoid the effect of ADP inhibition on ATP-hydrolysis activity. The bound nucleotide on each mutant $V_{1}$ was removed by successive Pi-EDTA treatment, as described earlier (Nakano et al, 2008). The ATP-hydrolysis activity of each mutant $V_{1}$ and $F_{1}$ were measured by the enzyme coupling assay (Yokoyama et al, 1998).

\section{Data deposition}

The atomic coordinates and the structure factors have been deposited in the Protein Data Bank for structure (PDB ID; 3GQB)

\section{Supplementary data}

Supplementary data are available at The EMBO Journal Online (http://www.embojournal.org).

\section{Acknowledgements}

We thank S Hayashi, T Ikegami and Y Suzuki for critical discussion, members of Hisabori and Yoshida laboratories, Diamond MPL and ICORP in Odaiba for help and advice, T Yano for laboratory management and encouragement. This work was partly supported by Grantsin-Aid from the Ministry of Education, Science, Sports and Culture of Japan to KY (No. 21023009 and 21370042), Targeted Proteins Research Program (TPRP) (B-37, to KY), Welcome Trust to MI, and a Cancer Institute of NSW fellowship (MJM). We thank a referee who suggested the possible twinning problem of the structure.

\section{Conflict of interest}

The authors declare that they have no conflict of interest.

\section{References}

Abrahams JP, Leslie AG, Lutter R, Walker JE (1994) Structure at 2.8 A resolution of F1-ATPase from bovine heart mitochondria. Nature 370: 621-628

Boyer PD (1993) The binding change mechanism for ATP synthase-some probabilities and possibilities. Biochim Biophys Acta 1140: 215-250

Brunger AT, Adams PD, Clore GM, DeLano WL, Gros P, GrosseKunstleve RW, Jiang JS, Kuszewski J, Nilges M, Pannu NS, Read RJ, Rice LM, Simonson T, Warren GL (1998) Crystallography \& NMR system: a new software suite for macromolecular structure determination. Acta Crystallogr D Biol Crystallogr 54: 905-921

Collaborative Computational Project, Number 4 (1994) 'The CCP4 Suite: Programs for Protein Crystallography'. Acta Cryst D50: 760-763

Dittrich M, Hayashi S, Schulten K (2004) ATP hydrolysis in the betaTP and betaDP catalytic sites of F1-ATPase. Biophys $J$ 87: $2954-2967$

Emsley P, Cowtan K (2004) Coot: model-building tools for molecular graphics. Acta Crystallogr D Biol Crystallogr 60: 2126-2132

Feng Y, Forgac M (1994) Inhibition of vacuolar H(+)-ATPase by disulfide bond formation between cysteine 254 and cysteine 532 in subunit A. J Biol Chem 269: 13224-13230

Forgac M (2007) Vacuolar ATPases: rotary proton pumps in physiology and pathophysiology. Nat Rev Mol Cell Biol 8: 917-929

Garin J, Vincon M, Gagnon J, Vignais P (1994) Photolabeling of mitochondrial F1-H + ATPase by 2-azido[3H]ADP and 8azido[3H]ADP entrapped as fluorometal complexes into the catalytic sites of the enzyme. Biochemistry 33: 3772-3777

Imamura H, Funamoto S, Yoshida M, Yokoyama K (2006) Reconstitution in vitro of V1 complex of Thermus thermophilus V-ATPase revealed that ATP binding to the A subunit is crucial for V1 formation. J Biol Chem 281: 38582-38591

Imamura H, Nakano M, Noji H, Muneyuki E, Ohkuma S, Yoshida M, Yokoyama K (2003) Evidence for rotation of V1-ATPase. Proc Natl Acad Sci USA 100: 2312-2315

Imamura $\mathrm{H}$, Takeda $\mathrm{M}$, Funamoto $\mathrm{S}$, Shimabukuro K, Yoshida $\mathrm{M}$ Yokoyama K (2005) Rotation scheme of V1-motor is different from that of F1-motor. Proc Natl Acad Sci USA 102: 17929-17933

Jones TA, Zou JY, Cowan SW, Kjeldgaard M (1991) Improved methods for building protein models in electron density maps and the location of errors in these models. Acta Crystallogr A 47 (Part 2): 110-119

Kabaleeswaran V, Puri N, Walker JE, Leslie AG, Mueller DM (2006) Novel features of the rotary catalytic mechanism revealed in the structure of yeast F1 ATPase. EMBO J 25: 5433-5442

Liu Q, Kane PM (1996) Mutational analysis of the catalytic subunit of the yeast vacuolar proton-translocating ATPase. Biochemistry 35: $10938-10948$

Maegawa Y, Morita H, Iyaguchi D, Yao M, Watanabe N, Tanaka I (2006) Structure of the catalytic nucleotide-binding subunit A of A-type ATP synthase from Pyrococcus horikoshii reveals a novel domain related to the peripheral stalk. Acta Crystallogr D Biol Crystallogr 62: 483-488

Manolson MF, Rea PA, Poole RJ (1985) Identification of 3-O-(4-benzoyl)benzoyladenosine $5^{\prime}$-triphosphate- and $N, N^{\prime}$ dicyclohexylcarbodiimide-binding subunits of a higher plant $\mathrm{H}+$-translocating tonoplast ATPase. $J$ Biol Chem 260: $12273-12279$

Matsui T, Muneyuki E, Honda M, Allison WS, Dou C, Yoshida M (1997) Catalytic activity of the alpha3beta3gamma complex of F1-ATPase without noncatalytic nucleotide binding site. J Biol Chem 272: 8215-8221

McCoy AJ, Grosse-Kunstleve RW, Adams PD, Winn MD, Storoni LC, Read RJ (2007) Phaser crystallographic software. J Appl Cryst 40: 658-674

Menz RI, Walker JE, Leslie AG (2001) Structure of bovine mitochondrial $F(1)$-ATPase with nucleotide bound to all three catalytic sites: implications for the mechanism of rotary catalysis. Cell 106: 331-341

Murshudov GN, Vagin AA, Dodson EJ (1997) Refinement of macromolecular structures by the maximum-likelihood method. Acta Crystallogr D Biol Crystallogr 53: 240-255

Nakano $M$, Imamura $H$, Toei $M$, Tamakoshi $M$, Yoshida $M$, Yokoyama K (2008) ATP hydrolysis and synthesis of a rotary motor V-ATPase from Thermus thermophilus. J Biol Chem 283: 20789-20796 
Olesen C, Picard M, Winther AM, Gyrup C, Morth JP, Oxvig C, Moller JV, Nissen P (2007) The structural basis of calcium transport by the calcium pump. Nature 450: 1036-1042

Otwinowski Z, Minor W (1997) Processing of X-ray diffraction data collected in oscillation mode. Methods Enzymol 276: 307-326

Painter J, Merritt EA (2006) A molecular viewer for the analyssiss of TLC rigid-body motion in macromolecules. Acta Cryst, D 62: $439-450$

Schafer IB, Bailer SM, Duser MG, Borsch M, Bernal RA, Stock D, Gruber G (2006) Crystal structure of the archaeal A1Ao ATP synthase subunit B from Methanosarcina mazei Gol: implications of nucleotide-binding differences in the major A1Ao subunits A and B. J Mol Biol 358: 725-740

Shao E, Nishi T, Kawasaki-Nishi S, Forgac M (2003) Mutational analysis of the non-homologous region of subunit $A$ of the yeast V-ATPase. J Biol Chem 278: 12985-12991

Terwilliger TC (2000) Maximum-likelihood density modification. Acta Crystallogr D Biol Crystallogr 56: 965-972

Tsutsumi S, Denda K, Yokoyama K, Oshima T, Date T, Yoshida M (1991) Molecular cloning of genes encoding major two subunits of a eubacterial V-type ATPase from Thermus thermophilus. Biochim Biophys Acta 1098: 13-20

Vasilyeva E, Forgac M (1996) 3'-O-(4-benzoyl)benzoyladenosine 5'triphosphate inhibits activity of the vacuolar $(\mathrm{H}+)$-ATPase from bovine brain clathrin-coated vesicles by modification of a rapidly exchangeable, noncatalytic nucleotide binding site on the $\mathrm{B}$ subunit. J Biol Chem 271: 12775-12782

Yamasaki K, Wang G, Daiho T, Danko S, Suzuki H (2008) Roles of Tyr122-hydrophobic cluster and $\mathrm{K}+$ binding in $\mathrm{Ca} 2+$-releasing process of ADP-insensitive phosphoenzyme of sarcoplasmic reticulum Ca2 + -ATPase. J Biol Chem 283: 29144-29155

Yokoyama K, Imamura H (2005) Rotation, structure, and classification of prokaryotic V-ATPase. J Bioenerg Biomembr 37: 405-410

Yokoyama K, Muneyuki E, Amano T, Mizutani S, Yoshida M, Ishida M, Ohkuma S (1998) V-ATPase of Thermus thermophilus is inactivated during ATP hydrolysis but can synthesize ATP. J Biol Chem 273: 20504-20510

Yokoyama K, Nagata K, Imamura $H$, Ohkuma S, Yoshida $M$, Tamakoshi M (2003) Subunit arrangement in V-ATPase from Thermus thermophilus. J Biol Chem 278: 42686-42691

Yokoyama K, Oshima T, Yoshida M (1990) Thermus thermophilus membrane-associated ATPase. Indication of a eubacterial V-type ATPase. J Biol Chem 265: 21946-21950

Yoshida M, Allison WS (1990) The ATPase activity of the alpha 3 beta 3 complex of the F1-ATPase of the thermophilic bacterium PS3 is inactivated on modification of tyrosine 307 in a single beta subunit by 7-chloro-4-nitrobenzofurazan. J Biol Chem 265: 2483-2487

Yoshida M, Muneyuki E, Hisabori T (2001) ATP synthase-a marvellous rotary engine of the cell. Nat Rev Mol Cell Biol 2: 669-677

The ЕМВо Journal is published by Nature SOWERाGHISRESERVED Publishing Group on behalf of European
Molecular Biology Organization. This article is licensed under a Creative Commons Attribution-NoncommercialShare Alike 3.0 Licence. [http://creativecommons.org/ licenses/by-nc-sa/3.0/] 